\title{
Research on Modeling and Simulation of Battery Swap Station Based on Discrete Event
}

\author{
Peng $\operatorname{Han}^{1,} \mathrm{a}^{*}$, Yue Zhang ${ }^{1, b}$ \\ ${ }^{1}$ School of Computer and Communication Engineering, Northeastern University at Qinhuangdao, \\ 066004, China. \\ a email: hp@mail.neuq.edu.cn, bemail: 710116781@qq.com
}

\section{Keywords: Smart Grid; Battery Swap Stations; Discrete Event Modeling; Simulation System}

\begin{abstract}
This paper builds a model for the operation of battery swap stations based on discrete event and build corresponding simulation system for optimized configuration of battery swap stations. The results showed that both the quantity of the robots and the utilization of source were important factors to ensure the optimal configuration of battery swap stations in the premise of supposing the traffic flow and the higher utilization of source, the higher operation efficiency in the premise of the normal operation of battery swap stations.
\end{abstract}

\section{Introduction}

With the development of world economy, ecological environment worsening and the contradiction between the growing demand for energy is increasingly obvious. And the development of electric vehicles has great influence in improving energy efficiency and cutting $\mathrm{CO} 2$ emissions [1-4].

In recent years, scholars at home and abroad according to the different characteristics of electrical changing station constructed many kinds of models. Liu Yujiao and other colleagues[5] reduce the electric car in plant operation as much as possible in the case of meeting the demand of electric vehicles in electricity and establish the mathematical model which makes expectation and variance of the cost as the objective function; Karbasioun M M and other colleagues[6] established markov decision process model under the premise of that charging vehicles obey the poison distribution and the charging demand are independent of each other; Wen-qi tian and other colleagues[7] according to the characteristics of the electric vehicle in plant, make charging power in different power plant each time as control object, establish the mathematical model of charging order scheduling policy for three kinds of objective function of Smooth the load fluctuation, poor peak valley, load rate,. In the simulation, Zhang Shu and other colleagues[8] introduced in power plant electric vehicles in traditional unit combination problem, established the new model of in power plant and power network interactive unit combination and called on the software of CPLEX to solve problems based on Matlab; Liu Nian and other colleagues[9] through the research of the active control strategy of power plant operation In the case of distribution network fault, built the simulation model of feeder automation based on PSCAD software and simulated analyze the influence for the feeder automation when power plants are using active control strategy.

Due to countries actively promote the development of electric buses, electric buses as infrastructure are used in a big number. Therefore, this paper will regard the operation of the electric bus in power plant as the research object. In view of the problem of optimal operation in power plant, considering the in plant operation more accord with the characteristics of discrete event system and the characteristics of the randomness, we proposed discrete event modeling method based on AnyLogic software platform with the simulation system of form a complete set of and find out the optimal configuration under the certain conditions in power station.

\section{Model of Electric buses in power station}

In the process of discrete event system modeling for electric vehicles in plant operation process, 
to stay in power electric cars as the "real" in the system, fully charged batteries as "resource" in the system, the charge in the electric process as a "service" in the system and all electric cars with no response as the "queue" in the system. The operating model of electric cars in power station is mainly composed of input and output variables, operation and some basic parameters [10]. The basic flow chart shown in Figure 1.

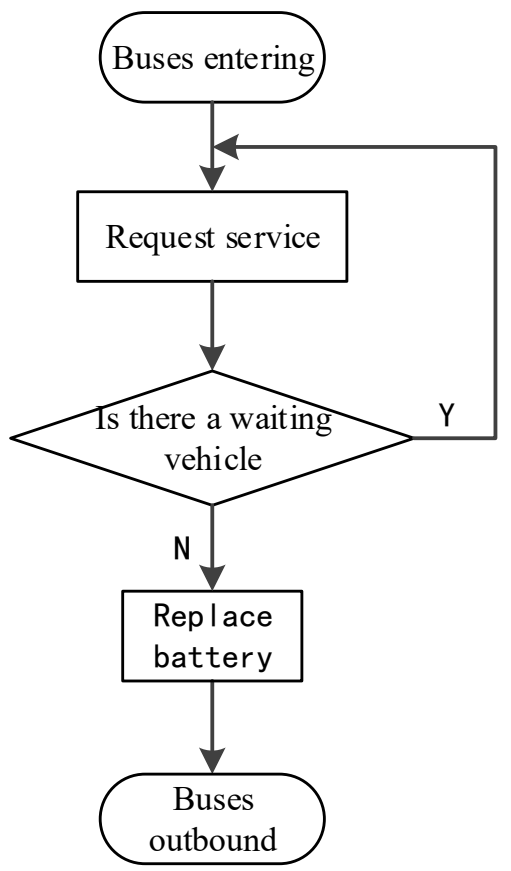

Fig.1. The basic flow chart

\section{The main operating of the model}

According to the actual situation, the main input of the electric car in plant operation model include the frequency and the rate of electric vehicles entering in power plant and the amount of electric cars, the main output is resource utilization.

The frequency of the electric vehicles entering in power station and the amount of electric cars determine the flow of electric vehicle in power plant. The facilities needed in power plant can be determine by the number of cars.

The frequency and rate of the electric vehicles entering in power station and the amount of electric cars determine the length of electric car in power station waiting queue as shown in figure 2 , which equal to the area of the waiting area.

Through collecting the resources utilization rate of electric vehicle in plant and finding out the number of the optimal electrical infrastructure under the condition of fixed flow and the maximum service traffic under the fixed number of infrastructure to optimize operation in power plant.

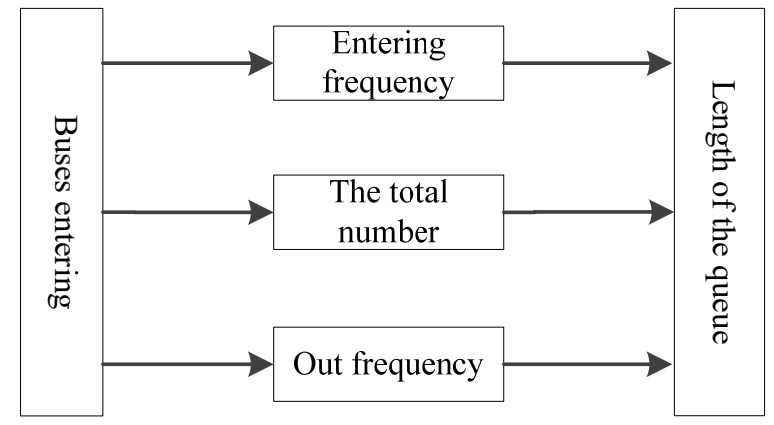

Fig.2. The key points for the length of waiting queue

The middle operation in plant operation model mainly includes electric car replacement, the collection of the replaced battery and battery charging, which shown in figure 3 . 
Electric cars battery replacement as a "service", has the delay time which is equal to the actual time for a power plant electric cars for electricity, usually 6 to 8 minutes. And the delay time is not set in the model because the work of the collection of the replaced battery and the replacement of the battery are at the same time in the actual situation. We need to discuss the work of charging respectively according to the different type battery charging time.

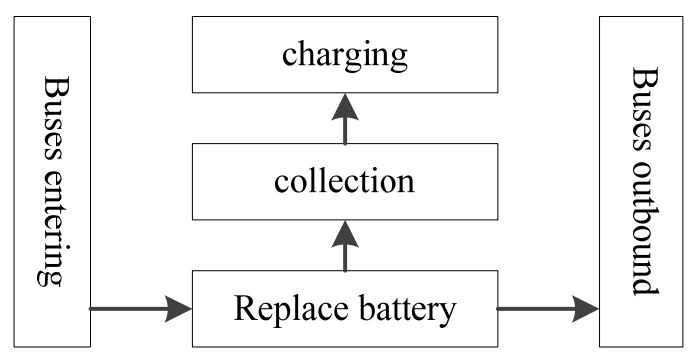

Fig.3. The middle operation of the model

\section{Model Construction}

The simulation program includes three parts, the input, intermediate operation which includes battery replaced and the separation and storage operations of the battery and output program, all the procedures as shown in figure 4.

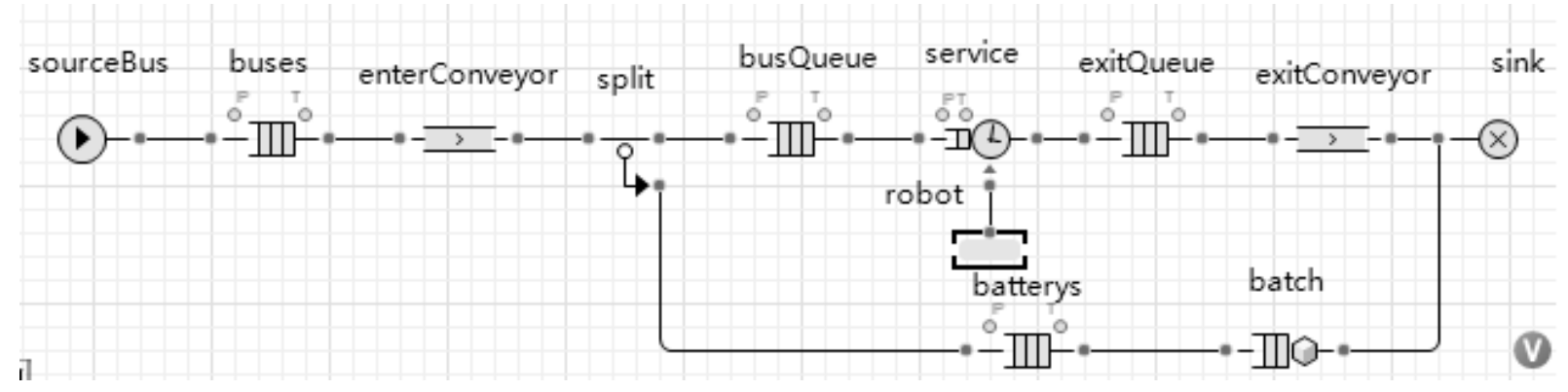

Fig.4. The procedures of the battery switching

Input program includes the Source object, Queue object and Conveyor object, which named sourceBus, buses and enterConveyor respectively in this case. The Source object is usually the starting point of the discrete event model and generated electric cars in this simulation. Queue entities simulated receiving the next object of a wait flow chart or the queue(cache) of the storage area, add the Queue object to store the electric car. Conveyor object move along a certain path at the speed of a given entity and ensure that the minimum interval between entities. It is used to ensure the space between the electric buses in this case.

Battery separation operation includes the split object which is named split in this case. The split object establishes one or more other entities for each incoming entities and output them through outCopy port. It is used to separate the battery in this case.

Battery storage operation includes Queue object and Batch object, which are named batterys and Batch respectively in this case. The Queue object is used to store the separation of the battery. Batch object not only can delete the original entity, establish a new entity and transform multiple entities into a single entity (beam), but also can add the original entity to the new entity to realize the temporary baling and recover in the subsequent Unbatch object's unbound operation. This case is used to pack away storage battery every ten.

In electrical operation include the Service object and ResourcePool object which are named Service and robot respectively in this case. Service object will occupy certain resources in the operation of entity, produce the operation delay and release the resources occupied after the operation completed. In this case, the Service object is used to simulate the change process and the delay time is set to 6 minutes. ResourcePool object defines a set of resources and the entities need to use resources to participate in a series of operations. ResourcePool object is used to represent the 
robot in electricity in power plant in this case.

Output program includes Conveyor and Sink object which named exitConveyor and Sink respectively in this case. Conveyor object represents the outbound path of the electric cars. Sink object is used to discard entity. Sink object represents electric buses out of power plant and pack out of the battery in this case.

\section{Analysis of the simulation}

This article sets the total number of electric buses are 200 and the average speed is $20 \mathrm{~km} / \mathrm{h}$. It sets the electric rate schedule in different traffic.

The resource utilization and the number of waiting buses are counted in different quantity of electrical robots. It is concluded the optimal allocation of resources under the set of the total number of electric buses.

Setting the number of electric robots according to the order of the 5,9 to simulate and through the analysis of the data from figure 5 we can see that: The resource utilization is close to 1 when the number of robots are 5, but the number of waiting buses are close to 15 in a period of one hour which causes seriously blocked. The number of waiting buses is close to 0 when the number of robots are 9, but the resource utilization is only one half which causes the waste of resource.
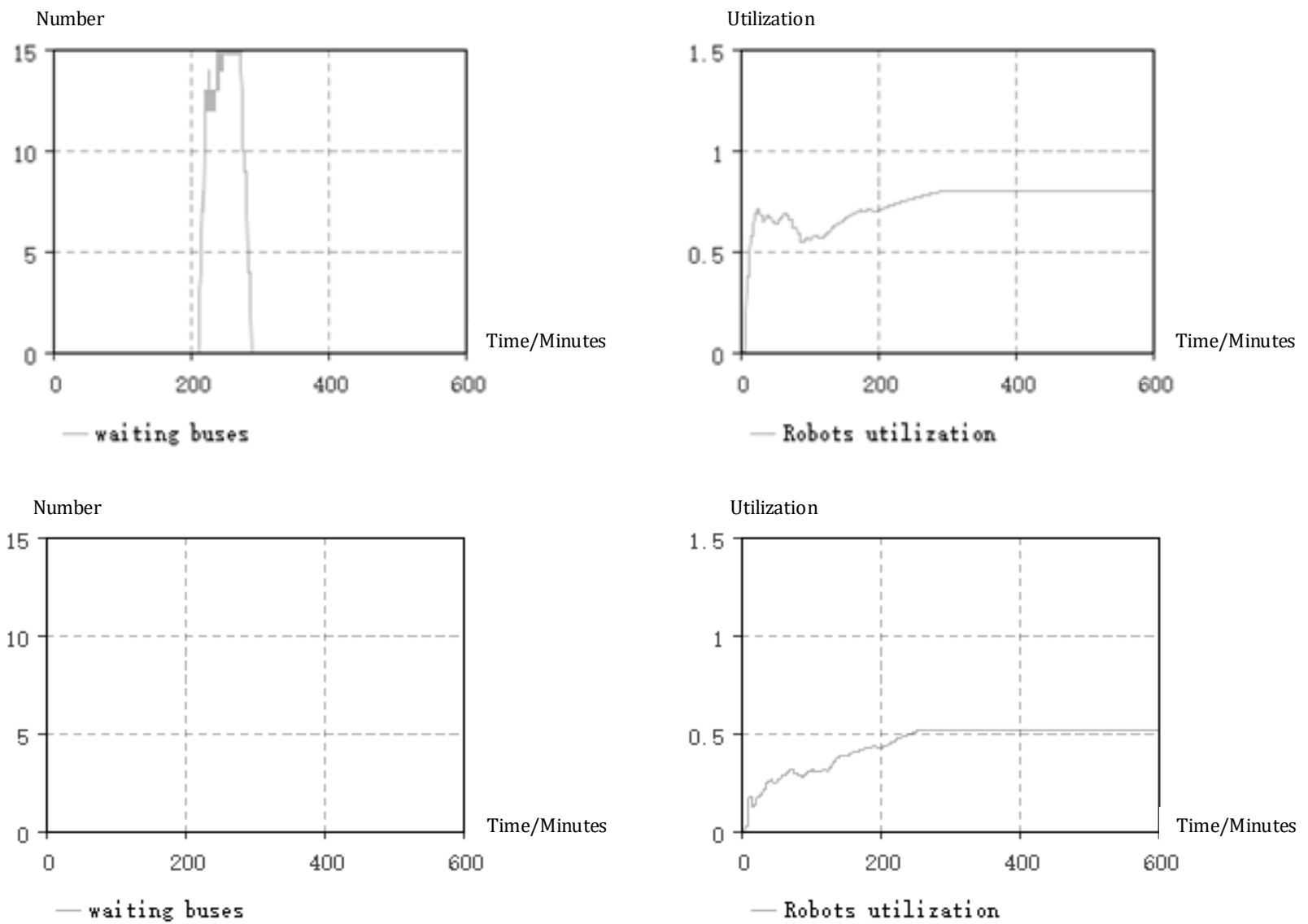

Fig.5. Average resource utilization and the number of waiting buses when the number of robots is set to 5 and 9

In conclusion, the number of electrical robots should be set up between 5 and 9 , set the number of the robot respectively for 6,7 and 8 with the enumeration method. Figure 6 shows that considering waiting time and resource utilization, get the larger resource utilization under the smaller number of waiting buses while the number of the robots is set to 7 , so the allocation of resources meets the condition of the optimum operation in plant. 

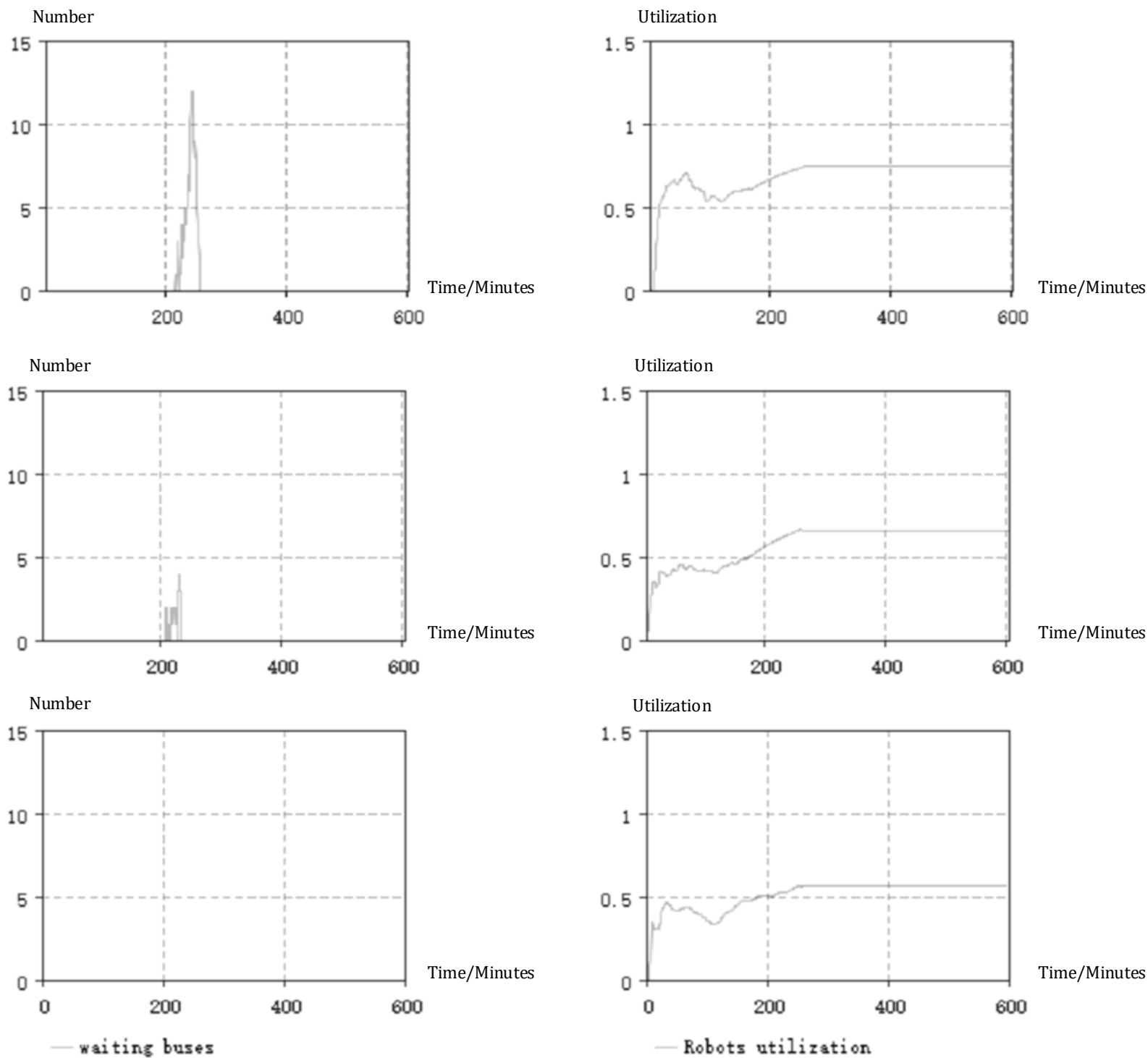

Fig.6. Average resource utilization and the number of waiting buses when the number of robots is set to 6,7 and 8

\section{Conclusion}

In this article, we regard the operation system of the electric vehicle in power station as the research object, through the analysis of the operation situation in power station, point out the influence factors of normal operation in power station, build the power station operation model based on discrete event, use AnyLogic simulation software for simulation model in the assumption of traffic flow in different time periods and verify and analyze the simulation results.

\section{Acknowledgement}

This work is supported by the National Natural Science Foundation of China (61374097 and 61473066), the Program for New Century Excellent Talents in University (NCET-12-0103), the Basic Science \& Research Fund of Northeastern University (N152303010) and of Northeastern University at Qinhuangdao (XNB201601), and the Science and Technology Research Project of Higher Education of Hebei Province (QN2016315). 


\section{References}

[1] Liangliang Chen, Hao Zhang, Feng Ni, et al. Present Simulation and Development Trend for Construction of Electric Vehicle Energy Supply Infrastructure [J]. Automation of Electric Power Systems, 2011, 35(14): 11-17.

[2] Anderson R N, Boulanger B, Powell W B, Scott W. Adaptive Stochastic Control for the Smart Grid [J]. Proceeding of the IEEE, 2011, 99(6): 1098-1115.

[3] Shaoyong Wang. Design and operation of micro-grid based on distributed generation [J]. Electric Power Automation Equipment, 2011, 31(4): 120-123.

[4] Wenliang Zhang, Bin Wu, Wufeng Li, et al. Discussion on Development Trend of Battery Electric Vehicles in China and Its Energy Supply Mode [J]. Power System Technology, 2009, 33(4): $1-5$.

[5] Yujiao Liu, Chuanwen Jiang, Xu Wang, er al. Energy management with stochastic constraint and multi-objective optimization algorithm for electric vehicle battery swap station [J]. Electric Power Automation Equipment, 2013, (8): 59-63+69.

[6] Karbasioun M M, Lambadaris I, Shaikhet G, et al. Optimal Charging Strategies for Electrical Vehicles under Real Time Pricing[C] // 2014 IEEE International Conference on Smart Grid Communications, 2014: 746-751.

[7] Wenqi Tian, Jinghan He, Jiuchun Jiang, et al. Research on dispatching strategy for coordinated charging of electric vehicle battery swapping station $[\mathrm{J}]$. Power System Protection and Control, 2012, 40(21): 114-119.

[8] Shu Zhang, Zechun Hu, Yonghua Song et al. Research on Unit Commitment Considering Interaction Between Battery Swapping Station and Power Grid [J]. Proceedings of the CSEE, 2012, 32(10): 8+49-55.

[9] Nian Liu, Yingda Zhang, Jing Zhao, et al. An Active Control Strategy for Vehicle to Grid Operation of Battery Swap Station Under Distribution Network Fault [J]. Power System Technology, 2014, 38(4): 925-932.

[10]Xing Wei, Ying Liu, Xiaoming Liu, et al. AnyLogic System Simulation Environment [C] // Signal and Information Processing Professional Committee of China Aviation Society, 2004: 4-4. 